\title{
Intravenous immunoglobulin in the therapy of adult acute fulminant myocarditis: A retrospective study
}

\author{
DAN-QING YU ${ }^{1 *}$, YING WANG ${ }^{2 *}$, GUI-ZHOU MA ${ }^{2 *}$, RONG-HE XU ${ }^{2 *}$, \\ ZHI-XIONG CAI ${ }^{2}$, CHU-MIN NI ${ }^{2}$, PING CHEN $^{2}$ and ZHI-DAN ZHU ${ }^{2}$ \\ ${ }^{1}$ Department of Coronary Care Unit, Guangdong Cardiovascular Institute, Guangdong Academy of Medical Science, \\ Guangdong General Hospital, Guangzhou, Guangdong 510080; ${ }^{2}$ Department of Cardiology, \\ Affiliated Shantou Hospital of Sun Yat-sen University, Jinping, Shantou, Guangdong 515031, P.R. China
}

Received June 8, 2013; Accepted September 30, 2013

DOI: $10.3892 /$ etm.2013.1372

\begin{abstract}
Acute fulminant myocarditis (AFM) is a serious heart disease with limited treatment. This observational retrospective study aimed to investigate whether intravenous immunoglobulin (IVIG) was able to improve left ventricular function and reduce the episodes of arrhythmia in adult patients with AFM. The medical records of all patients with AFM who were admitted to the Critical Care Unit of Guangdong General Hospital (Guangzhou, China) between January 2001 and December 2010 were reviewed. A cohort of 58 patients was included in the study. Of these 58,32 patients were treated with IVIG (400 mg/kg per day) for five days, while the remaining patients did not receive IVIG therapy. The patients who received IVIG therapy had a higher left ventricular ejection fraction (LVEF) and a reduced left ventricular end-diastolic diameter (LVDD) compared with the non-IVIG therapy patients four weeks subsequent to the treatment $\left(\mathrm{P}_{\mathrm{LVEF}}=0.011\right.$ and $\left.\mathrm{P}_{\mathrm{LVDD}}=0.048\right)$. The post-treatment incidence of ventricular tachycardia/ventricular fibrillation (VT/VF) and atrioventricular block (AVB) was reduced in the patients who received IVIG therapy compared with the baseline values $\left(\mathrm{P}_{\mathrm{VT} / \mathrm{VF}}=0.025, \mathrm{P}_{\mathrm{AVB}}=0.003\right)$; however, no significant differences were observed in the non-IVIG therapy patients $\left(\mathrm{P}_{\mathrm{VT} / \mathrm{VF}}=0.564, \mathrm{P}_{\mathrm{AVB}}=0.083\right)$ following treatment. There were two mortalities in the IVIG therapy group and seven in the non-IVIG therapy group $(\mathrm{P}=0.072)$. This retrospective study suggested that the use of IVIG for the treatment of AFM may
\end{abstract}

Correspondence to: Professor Dan-Qing Yu, Department of Coronary Care Unit, Guangdong Cardiovascular Institute, Guangdong Academy of Medical Science, Guangdong General Hospital, 96 Dongchuan Road, Guangzhou, Guangdong 510080, P.R. China

E-mail: gdydqprofessor@163.com

*Contributed equally

Key words: acute fulminant myocarditis, heart failure, intravenous immunoglobulin, left ventricular ejection fraction, arrhythmia be associated with improved left ventricular function and reduced episodes of fulminant arrhythmias.

\section{Introduction}

Acute fulminant myocarditis (AFM) is clinically and pathologically defined as inflammation of the myocardium, leading to the sudden onset of heart failure, arrhythmia, fulminant hemodynamic collapse and sudden mortality $(1,2)$. The initial severe presentation and favorable long-term prognosis of AFM is associated with left ventricular function (3). AFM involves serious arrhythmias, which occasionally have lethal consequences due to cardiac dysfunction (4).

Intravenous immunoglobulin (IVIG) has been used to treat inflammatory and autoimmune diseases $(5,6)$. Immune or autoimmune mechanisms may be involved in the pathogenesis of myocarditis (7). The administration of immunoglobulin was reported to be of clinical value against certain viral infections due to the neutralization of the virus, the blockade of Fc receptors and the neutralization of a microbial toxin $(5,6,8,9)$. Although the immunosuppressive therapy has yielded conflicting results in patients with acute myocarditis (10), previous studies demonstrated the effect of the IVIG in acute myocarditis $(9,11)$. The beneficial effect of IVIG on myocarditis was demonstrated in an animal study using polyclonal immunoglobulin (11). In the pediatric population, IVIG was associated with improved recovery of left ventricular function (9). However, few studies have specifically focused on IVIG for adult AFM. This retrospective study was performed to evaluate the effect of IVIG on the cardiac function and cardiac rhythm of adult patients with AFM.

\section{Methods}

Patients and study design. This was an observational retrospective case study of inpatients who presented with AFM in Guangdong General Hospital (Guangzhou, China) between January 2001 and December 2010. The patients were included according to the following criteria: Adult (age $>18$ years), acute-onset (duration $<3$ months) congestive heart failure and impaired left ventricular function following 
a recent viral illness. Patients with coronary artery disease, long-standing dilated cardiomyopathy, structural heart disease, systemic autoimmune disease, Kawasaki disease, the presence of active infection and other specific causes of acute cardiomyopathy were excluded. Data were collected through patient chart review. This study was approved by the ethics committee for clinical investigations of Guangdong General Hospital and informed consent was obtained from the patient's family.

Data collection. Clinical data and demographic information were collected by the review of the medical records of the enrolled patients. The patients were divided into IVIG therapy and non-IVIG therapy (control) groups. Blood samples were obtained to analyze myocardial enzymes, troponin, brain natriuretic peptide, C-reactive protein and erythrocyte sedimentation rate at baseline. Echocardiography, electrocardiography and 24-h ambulatory electrocardiography were performed prior to and following treatment. Gender, age, cardiac function classification, parameters of echocardiography, blood test data and incidence of complications were compared between the two groups.

Echocardiography. Echocardiography data were collected by previously reported methods (12). Left ventricular ejection fraction (LVEF), diameter of the left atrium (LA), left ventricular end-diastolic diameter (LVDD), left ventricular systolic diameter (LVDS), diameter of the right atrium (RA) and diameter of the right ventricle (RV) were measured using echocardiography. The data of left ventricle and left atrium were measured on parasternal long-axis view, and the data of right ventricle and right atrium were measured on apical four-chamber view. The recovery of left ventricular function was assessed in hospital and post-treatment (four weeks).

IVIG treatment regimens. IVIG (CSL Behring, Marburg, Germany) was administered at a dose of $400 \mathrm{mg} / \mathrm{kg}$ per day for five days. Other conventional therapies were administered as required, including high-dose vitamin $\mathrm{C}$, diuretics, digoxin, dopamine, dobutamine, angiotensin-converting enzyme inhibitor/angiotensin receptor blocker (ACEI/ARB), vasodilators (sodium nitroprusside, nitroglycerin) and glucocorticoids. Intra-Aortic Balloon Pumps (IABPs) were used to treat cardiogenic shock, while temporary pacemakers were applied to third-degree atrioventricular block (AVB).

Statistical analysis. The continuous variables that followed a symmetrical distribution are presented as the mean \pm standard deviation, with the exception of Fig. 1. The variables in Fig. 1 are presented as the mean \pm standard error. These continuous variables were compared using the two independent samples Student's t-test. Data that followed an asymmetric distribution are presented as medians and interquartile ranges, and were compared using the Kruskal-Wallis nonparametric test. The categorical variables are presented as frequencies and were analyzed using $\chi^{2}$ and Fischer exact tests. $\mathrm{P}<0.05$ was considered to indicate a statistically significant difference. All statistical analyses were performed with the Statistical Package for Social Sciences version 16.0 (SPSS Inc., Chicago, IL, USA).

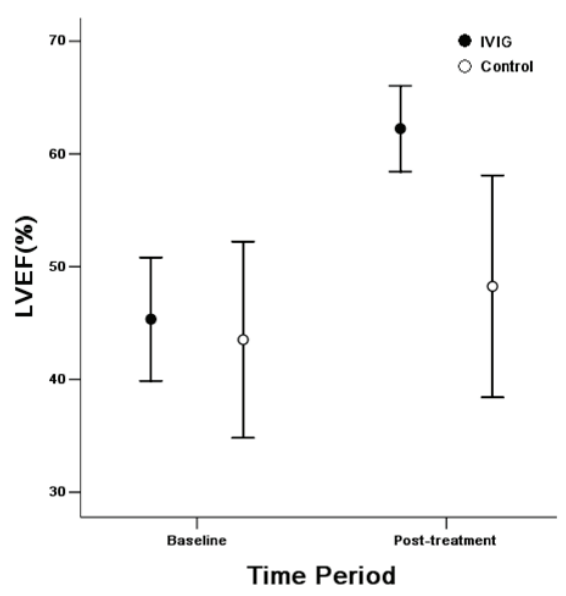

Figure 1. Left ventricular ejection fraction (LVEF; mean \pm standard error of the mean) in the intravenous immunoglobulin (IVIG) and control groups at baseline and post-treatment (4 weeks). There was no difference between the two groups in the mean LVEF at baseline $(\mathrm{P}=0.703)$. Post-treatment, the LVEF of the IVIG group was higher than that of the control group $(\mathrm{P}=0.011)$.

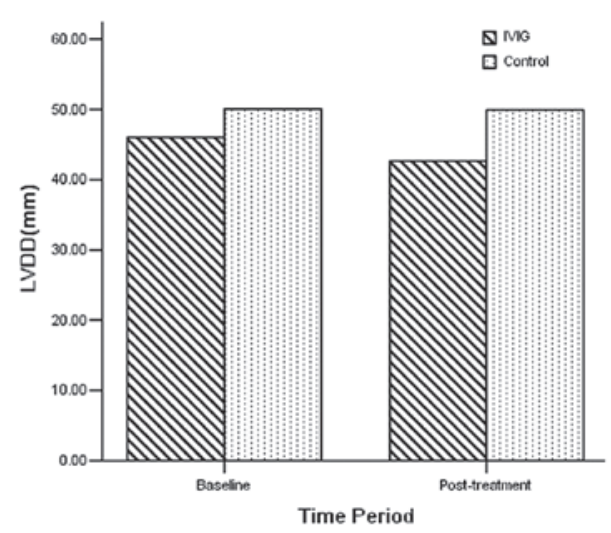

Figure 2. Left ventricular end-diastolic diameter (LVDD; mean \pm standard deviation) in the intravenous immunoglobulin (IVIG) and control groups at baseline and post-treatment (4 weeks). There was no difference between the two groups in the mean LVDD at baseline. Four weeks subsequent to treatment, the LVDD of the IVIG group was reduced compared with that of the control group $(\mathrm{P}=0.048)$.

\section{Results}

Baseline characteristics. A total of 75 patients were initially eligible for the study; however, 17 patients were excluded due to insufficient clinical data. Fifty-eight patients were ultimately included. Of these 58, 32 patients were treated with IVIG (400 mg/kg per day) for five days, while the remaining patients did not receive IVIG therapy. The IVIG and non-IVIG groups were similar with regard to baseline characteristics, including gender, age, cardiac function classification, parameters of echocardiography, blood test data and incidence of complications (Table I). The cardiac functions of the patients were classified as NYHA classes II to IV. Ten patients (33\%) of the IVIG group who had NYHA class IV heart failure or cardiogenic shock received IABP support, compared with three patients $(16 \%)$ in the control group $(\mathrm{P}=0.073)$. There were seven (27\%) mortalities in the control group and two $(6 \%)$ in the IVIG group ( $\mathrm{P}=0.072)$. 
Table I. Initial demographic and clinical characteristics of 58 patients with AFM.

\begin{tabular}{|c|c|c|c|}
\hline Variable & IVIG group $(\mathrm{n}=32)$ & Control group $(n=26)$ & P-value \\
\hline Age (years) ${ }^{\mathrm{a}}$ & $30.6 \pm 14.1$ & $30.0 \pm 16.6$ & 0.905 \\
\hline Male $^{\mathrm{b}}$ & $15 / 32(47)$ & $13 / 26(50)$ & 0.813 \\
\hline NYHA classification $^{\mathrm{b}}$ & & & 0.144 \\
\hline Class I & $0 / 32(0)$ & $0 / 26(0)$ & \\
\hline Class II & $8 / 32(25)$ & $6 / 26(23)$ & \\
\hline Class III & $13 / 32(41)$ & $11 / 26(42)$ & \\
\hline Class IV & $11 / 32(34)$ & $9 / 26(35)$ & \\
\hline \multicolumn{4}{|l|}{ Echocardiography ${ }^{\mathrm{a}}$} \\
\hline Left ventricular ejection fraction (\%) & $45.3 \pm 15.2$ & $43.5 \pm 19.6$ & 0.703 \\
\hline Diameter of left atrium (mm) & $30.3 \pm 5.3$ & $31.5 \pm 7.3$ & 0.711 \\
\hline Diameter of right atrium (mm) & $43.0 \pm 8.7$ & $46.1 \pm 9.0$ & 0.211 \\
\hline Left ventricular diastolic diameter (mm) & $47.1 \pm 7.3$ & $50.1 \pm 9.3$ & 0.197 \\
\hline Left ventricular systolic diameter (mm) & $35.3 \pm 8.9$ & $37.9 \pm 12.2$ & 0.543 \\
\hline Diameter of right ventricle (mm) & $50.9 \pm 7.7$ & $50.5 \pm 13.7$ & 0.898 \\
\hline \multicolumn{4}{|l|}{ Laboratory tests ${ }^{\mathrm{a}}$} \\
\hline A-hydroxybutyrate dehydrogenase (U/l) & $959.2 \pm 1167.6$ & $641.8 \pm 486.8$ & 0.585 \\
\hline Lactate dehydrogenase (U/1) & $1707.7 \pm 3021.5$ & $923.6 \pm 851.9$ & 0.632 \\
\hline Creatine kinase (U/l) & $2228.3 \pm 5271.8$ & $889.1 \pm 825.7$ & 0.179 \\
\hline Creatine kinase-MB (U/l) & $40.4 \pm 56.2$ & $28.8 \pm 23.9$ & 0.454 \\
\hline Troponin $(\mathrm{ng} / \mathrm{ml})$ & $10.2 \pm 21.8$ & $2.2 \pm 2.7$ & 0.789 \\
\hline Brain natriuretic peptide (pg/ml) & $12746.8 \pm 13620.1$ & $4690.6 \pm 8780.7$ & 0.117 \\
\hline C-reactive protein $(\mathrm{mg} / \mathrm{l})$ & $46.5 \pm 51.2$ & $34.9 \pm 31.7$ & 0.480 \\
\hline Erythrocyte sedimentation rate $(\mathrm{mm} / \mathrm{h})$ & $20.0 \pm 16.5$ & $19.7 \pm 18.8$ & 0.969 \\
\hline \multicolumn{4}{|l|}{ Complications $^{\mathrm{b}}$} \\
\hline Pulmonary edema & $13 / 32(41)$ & $7 / 26(27)$ & 0.428 \\
\hline Cardiogenic shock & $12 / 32(38)$ & $4 / 26(15)$ & 0.061 \\
\hline Multiple Organ Dysfunction Syndrome & $8 / 32(25)$ & $2 / 26(8)$ & 0.166 \\
\hline Renal failure & $8 / 32(25)$ & $1 / 26(4)$ & 0.065 \\
\hline Cardiac arrest & $1 / 32(3)$ & $2 / 26(8)$ & 0.853 \\
\hline Aspen syndrome & $2 / 32(6)$ & $4 / 26(15)$ & 0.482 \\
\hline \multicolumn{4}{|l|}{ Therapies $^{\mathrm{b}}$} \\
\hline Vitamin C & 30/32 (94) & 23/26 (88) & 0.808 \\
\hline Glucocorticoid & $28 / 30(93)^{\mathrm{c}}$ & $18 / 19(95)^{\mathrm{c}}$ & 0.088 \\
\hline Intra-aortic balloon pumps & $10 / 30(33)^{c}$ & $3 / 19(16)^{c}$ & 0.073 \\
\hline Pacemaker & $15 / 30(50)^{\mathrm{c}}$ & $8 / 19(42)^{c}$ & 0.212 \\
\hline Mortality $^{\mathrm{b}}$ & $2 / 32(6)$ & $7 / 26(27)$ & 0.072 \\
\hline
\end{tabular}

${ }^{\text {a}}$ Values are presented as the mean \pm standard deviation, the data of left ventricle and left atrium were measured on parasternal long-axis view,

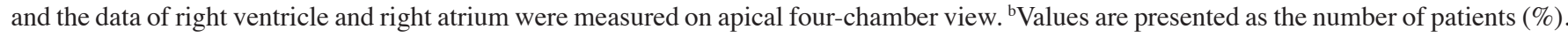
${ }^{c}$ Values are presented as the number of remaining patients following mortalities. IVIG, intravenous immunoglobulin; AFM, acute fulminant myocarditis; NYHA, New York Heart Association.

Changes in left ventricular function. The patients of the two groups did not differ significantly with regard to the echocardiographic data at the baseline. The effect of IVIG treatment on LVEF (Fig. 1) and LVDD (Fig. 2) was investigated.

At the baseline, the LVEF of the two groups was similar (IVIG versus control group: $45.3 \pm 15.2$ versus $43.5 \pm 19.6 \%$; $\mathrm{P}=0.703)$. Four weeks subsequent to treatment, the mean LVEF in the IVIG group was $62.2 \pm 10.2 \%$, compared with $48.3 \pm 20.4 \%$ in the control group. At four weeks post-treatment, the LVEF of the two groups had improved significantly compared with the baseline values $\left(\mathrm{P}_{\mathrm{IVIG}}<0.001, \mathrm{P}_{\text {control }}=0.027\right)$. The patients treated with IVIG had a higher LVEF than those without IVIG at four weeks $(\mathrm{P}=0.011)$.

Four weeks subsequent to treatment, the LVDD of the IVIG group $(44.2 \pm 5.8 \mathrm{~mm})$ was reduced compared with that of the control group $(49.6 \pm 10.3 \mathrm{~mm} ; \mathrm{P}=0.048$; Fig. 2). Furthermore, 

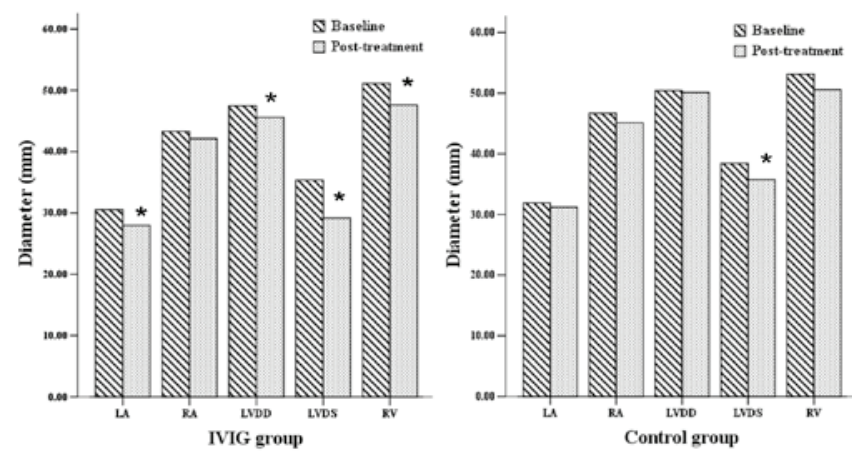

Figure 3. Echocardiography data (mean \pm standard deviation) of the intravenous immunoglobulin (IVIG) and control groups at baseline and posttreatment (4 weeks). The diameter of the left atrium (LA), left ventricular end-diastolic diameter (LVDD), left ventricular systolic diameter (LVDS) and diameter of right ventricle (RV) of the IVIG group diminished posttreatment. The diameter of LVDS of the control group diminished. The data of left ventricle (LV) and LA were measured on parasternal long-axis view, and the data of RV and right atrium (RA) were measured on apical fourchamber view. " $\mathrm{P}<0.05$ vs. baseline value.

the LA, LVDD, LVDS and RV of the IVIG group showed recovery at four weeks $\left(\mathrm{P}_{\mathrm{LA}}<0.001, \mathrm{P}_{\mathrm{LVDD}}=0.006, \mathrm{P}_{\mathrm{LVDS}}<0.001\right.$, $\left.\mathrm{P}_{\mathrm{RV}}=0.007\right)$, with the exception of RA ( $\left.\mathrm{P}=0.232\right)$. However, only the LVDS of the control group had improved at four weeks $\left(\mathrm{P}_{\mathrm{LVDS}}=0.012\right.$; Fig. 3).

Arrhythmia and changes in echocardiography results. The patients exhibited several types of arrhythmia, including bradycardia, AVB, atrial arrhythmia, ventricular arrhythmia and bundle branch block. The changes in the ST segment and $\mathrm{T}$ wave were also observed. Ventricular tachycardia/ventricular fibrillation (VT/VF) occurred in 8 of the 32 (25.0\%) patients in the IVIG group and 3 of the $26(11.5 \%)$ patients in the control group at baseline. Seventeen of the $32(53.1 \%)$ patients in the IVIG group exhibited AVB at baseline compared with 8 of the 26 (30.8\%) patients in the control group. Following treatment, the episodes of VT/VF and AVB were reduced in the IVIG group $\left(\mathrm{P}_{\mathrm{VT} / \mathrm{VF}}=0.025, \mathrm{P}_{\mathrm{AVB}}=0.003\right)$; however, there were no significant differences in the control group following treatment $\left(\mathrm{P}_{\mathrm{VT} / \mathrm{VF}}=0.564, \mathrm{P}_{\mathrm{AVB}}=0.083\right)$. Although the episodes of bradycardia, atrial arrhythmia, bundle branch block and the change in the ST-T tended to decrease post-treatment, the difference did not achieve statistical significance in this small sample (Table II).

\section{Discussion}

Despite the therapeutic efficacy suggested by previous studies $(9,11)$, IVIG therapy for AFM has been rarely reported. In this study, IVIG therapy improved the LVEF and reduced the LVDD compared with the control group. Furthermore, compared with the baseline values, the LA, LVDD, LVDS, RV and the episodes of VT/VF and AVB were improved at the post-treatment time-point in the IVIG group.

The damage to the myocardium in AFM may be mediated by predominantly immunological mechanisms rather than by the direct effect of viral infection and replication $(13,14)$. Previous studies have indicated the therapeutic effects of the 
IVIG in acute myocarditis $(11,15)$. Weller et al (11) observed that mouse polyclonal immunoglobulin minimized myocardial damage in Balb/c male mice infected intraperitoneally with coxsackievirus B3 when administered 24 or $48 \mathrm{~h}$ subsequent to infection (11). In another murine model of viral myocarditis, IVIG administration reduced the extent of myocardial necrosis or interstitial fibrosis and improved ventricular remodeling (15). In addition, certain clinical trials have indicated that IVIG may be beneficial in acute myocarditis. Drucker et al (9) suggested that the use of high-dose IVIG for the treatment of acute myocarditis was associated with the improved recovery of left ventricular function and with a improved survival rate during the first year following presentation (9). In patients with new-onset dilated cardiomyopathy treated with high-dose IVIG, LVEF improved by $17 \mathrm{EF}$ units (16). IVIG induced a significant increase in LVEF from $26 \pm 2$ to $31 \pm 3 \%$ in 40 patients with symptomatic chronic congestive heart failure (CHF) and LVEF of $<40 \%$ (17). A favorable clinical response was also observed in 10 case studies (18-27) and two case series $(28,29)$. However, according to a recent systematic review $(30)$, certain studies $(31,32)$ showed no benefit of IVIG. A randomized controlled trial (RCT) suggested that IVIG did not augment the improvement in LVEF for 62 patients with recent-onset dilated cardiomyopathy. This RCT showed no benefit with respect to cardiac function, functional outcome or event-free survival (33).

Certain studies have suggested the mechanism underlying the effect of high-dose IVIG in acute myocarditis (17,34-36). IVIG induced a marked rise in plasma levels of the anti-inflammatory mediators interleukin (IL)-10, IL-1 receptor antagonist and soluble tumor necrosis factor receptors. Furthermore, levels of N-terminal pro-atrial natriuretic peptide continued to decrease toward the end of the study during IVIG therapy (17). IVIG decreased cardiac inflammation and downregulated proinflammatory cytokines that have direct negative inotropic effects (34-36).

IVIG decreases the incidence of VT/VF and AVB in AFM in the present study. The combination of arrhythmias, including $\mathrm{VT} / \mathrm{VF}$ and $\mathrm{AVB}$, and heart failure presents a serious challenge in the management of AFM (1-4). The arrhythmias were resolved during the convalescent and remote phases among the surviving patients, and no atrial or ventricular arrhythmias were induced by the programmed stimulation (4).

In conclusion, this study suggested that IVIG for the treatment of AFM may be associated with improved recovery of left ventricular function and a reduction in the episodes of fulminant arrhythmias.

\section{References}

1. Magnani JW and Dec GW: Myocarditis: current trends in diagnosis and treatment. Circulation 113: 876-890, 2006.

2. Feldman AM and McNamara D: Myocarditis. N Engl J Med 343: 1388-1398, 2000.

3. Amabile N, Fraisse A, Bouvenot J, et al: Outcome of acute fulminant myocarditis in children. Heart 92: 1269-1273, 2006.

4. Ichikawa R, Sumitomo N, Komori A, et al: The follow-up evaluation of electrocardiogram and arrhythmias in children with fulminant myocarditis. Circ J 75: 932-938, 2011.

5. Wolf HM and Eibl MM: Immunomodulatory effect of immunoglobulins. Clin Exp Rheumatol 14 (Suppl 15): S17-S25, 1996.

6. Rosen FS: Putative mechanisms of the effect of intravenous gamma-globulin. Clin Immunol Immunopathol 67: S41-S43, 1993.
7. Kawai C: From myocarditis to cardiomyopathy: mechanisms of inflammation and cell death: learning from the past for the future. Circulation 99: 1091-1100, 1999.

8. Yu Z and Lennon VA: Mechanism of intravenous immune globulin therapy in antibody-mediated autoimmune diseases. $\mathrm{N}$ Engl J Med 340: 227-228, 1999.

9. Drucker NA, Colan SD, Lewis AB, et al: Gamma-globulin treatment of acute myocarditis in the pediatric population. Circulation 89: 252-257, 1994.

10. Mason JW, O'Connell JB, Herskowitz A, et al: A clinical trial of immunosuppressive therapy for myocarditis. The Myocarditis Treatment Trial Investigators. N Engl J Med 333: 269-275, 1995.

11. Weller AH, Hall M and Huber SA: Polyclonal immunoglobulin therapy protects against cardiac damage in experimental coxsackievirus-induced myocarditis. Eur Heart J 13: 115-119, 1992.

12. Newburger JW, Sanders SP, Burns JC, et al: Left ventricular contractility and function in Kawasaki syndrome. Effect of intravenous gamma-globulin. Circulation 79: 1237-1246, 1989.

13. O'Connell JB, Robinson JA, Henkin RE and Gunnar RM: Immunosuppressive therapy in patients with congestive cardiomyopathy and myocardial uptake of gallium-67. Circulation 64: 780-786, 1981.

14. AndersonJL,FowlesRE,UnverferthDV,etal:Immunosuppressive therapy of myocardial inflammatory disease. Initial experience and future trials to define indications for therapy. Eur Heart J 8 (Suppl): 263-266, 1987.

15. Kishimoto C, Takamatsu N, Kawamata H, et al: Immunoglobulin treatment ameliorates murine myocarditis associated with reduction of neurohumoral activity and improvement of extracellular matrix change. J Am Coll Cardiol 36: 1979-1984, 2000.

16. McNamara DM, Rosenblum WD, et al: Intravenous immune globulin in the therapy of myocarditis and acute cardiomyopathy. Circulation 95: 2476-2478, 1997.

17. Gullestad L, Aass H, Fjeld JG, et al: Immunomodulating therapy with intravenous immunoglobulin in patients with chronic heart failure. Circulation 103: 220-225, 2001.

18. Takeda Y, Yasuda S, Miyazaki S, et al: High-dose immunoglobulin $\mathrm{G}$ therapy for fulminant myocarditis. Jap Circ J 62: 871-872, 1998.

19. Nigro G, Bastianon V, Colloridi V, et al: Human parvovirus B19 infection in infancy associated with acute and chronic lymphocytic myocarditis and high cytokine levels: report of 3 cases and review. Clin Infect Dis 31: 65-69, 2000.

20. Tsai YG, Ou TY, Wang CC, et al: Intravenous gamma-globulin therapy in myocarditis complicated with complete heart block: report of one case. Acta Paediatr Taiwan 42: 311-313, 2001.

21. Shioji K, Matsuura Y, Iwase T, et al: Successful immunoglobulin treatment for fulminant myocarditis and serial analysis of serum thiredoxin: a case report. Circ J 66: 977-980, 2002

22. Tedeschi A, Airaghi L, Giannini S, et al: High-dose intravenous immunoglobulin in the treatment of acute myocarditis. A case report and review of the literature. J Intern Med 251: 169-173, 2002.

23. Stouffer GA, Sheahan RG, Lenihan DJ, et al: The current status of immune modulating therapy for myocarditis: a case of acute parvovirus myocarditis treated with intravenous immunoglobulin. Am J Med Sci 326: 369-374, 2003.

24. Kim HS, Sohn S, Park JY and Seo JW: Fulminant myocarditis successfully treated with high-dose immunoglobulin. Int $\mathrm{J}$ Cardiol 96: 485-486, 2004.

25. Braun JP, Schneider M, Dohmen P and Döpfmer U: Successful treatment of dilative cardiomyopathy in a 12-year-old girl using the calcium sensitizer levosimendan after weaning from mechanical biventricular assist support. J Cardiothorac Vasc Anest 18: 772-774, 2004.

26. Abe S, Okura Y, Hoyano M, et al: Plasma concentrations of cytokines and neurohumoral factors in a case of fulminant myocarditis successfully treated with intravenous immunoglobulin and percutaneous cardiopulmonary support. Circ J 68: 1223-1226, 2004.

27. English RF, Janosly JE, Ettedgui JA and Webber SA: Outcomes for children with acute myocarditis. Cardiol Young 14: 488-493, 2004.

28. Karaaslan S, Oran B, Caliskan U, et al: Hemolysis after administration of high-dose immunoglobulin in a patient with myocarditis. Turk J Haematol 20: 237-240, 2003.

29. Khan MA, Das B, Lohe A and Sharma J: Neonatal myocarditis presenting as an apparent life threatening event. Clin Pediatr (Phila) 42: 649-652, 2003. 
30. Robinson JL, Hartling L, Crumley E, et al: A systematic review of intravenous gamma globulin for therapy of acute myocarditis. BMC Cardiovascular Disorders 5: 12, 2005.

31. Kishimoto C, Shioji K, Kinoshita M, et al: Treatment of acute inflammatory cardiomyopathy with intravenous immunoglobulin ameliorates left ventricular function associated with suppression of inflammatory cytokines and decreased oxidative stress. Intern J Cardiol 91: 173-178, 2003.

32. Wang CY, Li Lu F, Wu MH, et al: Fatal coxsackievirus A16 infection. Pediatr Infect Dis J 23: 275-276, 2004.

33. McNamara DM, Holubkov R, Starling RC, et al: Controlled trial of intravenous immune globulin in recent-onset dilated cardiomyopathy. Circulation 103: 2254-2259, 2001
34. Finkel MS, Oddis CV, Jacob TD, et al: Negative inotropic effects of cytokines on the heart mediated by nitric oxide. Science 257: 387-389, 1992.

35. Leung DY, Burns JC, Newburger JW and Geha RS: Reversal of lymphocyte activation in vivo in Kawasaki syndrome by intravenous gammaglobulin. J Clin Invest 79: 468-472, 1987.

36. Hosenpud JD, Campbell SM and Mendelson DJ: Interleukin-1-induced myocardial depression in an isolate beating heart preparation. J Heart Transplant 8: 460-464, 1989. 